\title{
E scolha do br aço como sítio deimplantação do cateter venoso central de longa permanência em crianças: experiência do Serviço de Cirurgia Pediátrica do Hospital do Câncer Hospital do Câncer I - INCA - Rio de J aneiro
}

\author{
U se of the arm as long permanence central venous cateter insertion site in children: \\ experience of the pediatric surgery department at Instituto Nacional de Câncer - \\ Rio de J aneiro
}

\author{
Alberto Ribeiro Gonçalves, ${ }^{1}$ Carlos Humberto Vicuña Mariño, ${ }^{2}$ Ricardo Vianna de Carvalho, ${ }^{2}$ Renato van Wilpe, ${ }^{3}$ Simone de Oliveira Coelho, ${ }^{4}$ \\ Francisca Norma Albuquerque Gutierrez, ${ }^{4}$ Rosângela Finóquio, ${ }^{5}$ Marcelo Schimer ${ }^{6}$
}

\begin{abstract}
Resumo
O bjetivo: relatar nossa experiência com a colocação e 0 uso do cateter venoso central de longa permanência (CVCLP), implantado através das veias do braço. Pacientes e M étodos: trata-se de um trabalho retrospectivo em que foram analisados 586 cateteres implantados em crianças com câncer, de janeiro de 1995 a dezembro de 1999, no HC I - IN CA. Resultados: dos 586 CVCLP implantados em crianças com câncer, 91,5\% foram inseridos através das veias do braço (536), 45 (8\%) implantados no tórax e 5 (1\%) em outros sítios. As veias de escolha para 0 implante do cateter no braço foram as braquiais. 0 s resultados em relação às complicações (infecção, obstrução, exteriorização do anel fixador, ruptura do cateter, migração, entre outras), foram similares àqueles da literatura quando implantados no tórax. Conclusão: recomendamos a colocação do cateter no braço como primeira opção para colocação de cateteres venosos centrais de longa permanência para crianças com câncer, uma vez que mostrouse mais seguro, confortável, esteticamente mais tolerável e permitiu maior mobilidade aos pacientes, quando em uso do cateter do que os implantados no tórax. Implicação clínica: propomos a opção da colocação do CV CLP no braço como a primeira escolha por acharmos ser mais segura, mais confortável e eficiente, do que os implantados no tórax, para as crianças com câncer, que necessitem de um acesso venoso por tempo prolongado.
\end{abstract}

Palavras-chave: Cateter venoso central; $C$ ateter de longa permanência; Criança; O ncologia Pediátrica.

\footnotetext{
${ }^{1}$ Cirurgião Pediátrico, chefe do Serviço de Cirurgia Pediátrica do IN CA - H ospital do Câncer I

${ }^{2}$ Cirurgião Pediátrico, titular do Serviço de Cirurgia Pediátrica do INCA - H ospital do Câncer I

${ }^{3}$ Cirurgião Pediátrico, fellow do Serviço de Cirurgia Pediátrica do IN CA - Hospital do Câncer I

${ }^{4}$ Pós-graduanda, Serviço de Cirurgia Pediátrica do IN CA - H ospital do Câncer I.

${ }^{5}$ Enfermeira O ncológica, responsável pelo Ambulatório de Cateter Pediátrico do IN CA - H ospital do Câncer I.

${ }^{6}$ M édico Infectologista, titular da Comissão de Controle de Infecção H ospitalar do IN CA - H ospital do Câncer I.

Endereço para correspondência: Serviço de Cirurgia Pediátrica - Instituto Nacional de Câncer - Pça. da Cruz Vermelha, 23 - 20230-130 - Rio de Janeiro - RJ. E-mail:albertog@inca.gov.br
} 


\begin{abstract}
O bjective: To report our experience with insertion and use of LTCVC in the arm. Pacients and M ethods: It is a retrospective study. We analyzed 586 LTCVC inserted on children with cancer, from January 1995 to D ecember 1999. Results: From a total of 586 inserted LTCVC; 536 (91,5\%) were in the arm, 45 in the thorax and 5 were inserted in other sites. The vein of choice for the arm was the brachial vein, on its proximal third. All LTCVC were inserted at operating rooms, under general anesthesia. The tip of the catheter was localized on superior cava vein under fluoroscopy. O ur experience with the site of insertion on the arm had demonstrated that this site is safe, comfortable, esthetically better, and that it allows more mobility to the patients when it is activated. The results regarding infection rates for arm insertionare similar to those found on the literature for LTCVC thorax inserted. Conclusion: We indicate the arm (brachial vein) as the site of choice for inserting LTCVC for children with cancer. Clinical Implication: The insertion of LTCVC in the arm is a safe and efficient option for inserting LTCVC in children with cancer.
\end{abstract}

Key words: Central venous catheter; Long permanence catheter; Child; Pediatric O ncology.

\section{INTRO DUÇÃO}

A cateterização venosa central tem sido usada largamente em pacientes graves e/ou cronicamente doentes desde sua primeira descrição em 1952 por Aubaniac. ${ }^{1}$ As técnicas de inserção de cateteres, os sítios de flebotomia ou de punção, e os tipos de cateteres utilizados têm sido modificados com a evolução e com os avanços da bio-engenharia. Este desenvolvimento propiciou o uso crescente desses cateteres venosos centrais em crianças, nos últimos 20 anos. $\mathrm{H}$ oje eles são implantados de forma segura e promovem um acesso seguro e indolor de longo prazo ao sistema venoso central. ${ }^{2}$ As indicações principais para o seu uso são: administração de quimioterapia, nutrição parenteral, hemoderivados einfusão de medicamentos (antibióticos, analgésicos), além de permitirem a coleta de sangue.

São poucosos trabalhosquemostram o membro superior como via de escolha para a colocação de CVCLP. ${ }^{3-6}$

Acreditamos que a implantação de cateteres venosos centrais de longa permanência (CVCLP) no braço, através de flebotomia das veias braquiais ou axilares apresentam vantagens em relação à sua implantação no tórax. Este trabalho tem como objetivo demonstrar os motivos que nos levaram a considerar a implantação do CVCLP no braço como a primeira opção, sejam eles totalmente ou semi-implantados, em pacientes pediátricos.

0 local de implante tradicionalmente utilizado para os CV C LP é o tórax através da flebotomia ou da punção das veias jugulares esternas e internas e as veias subclávias.

Um local de exceção, quando todos os outros sítios já foram esgotados, são os membros inferiores (coxa), através dos acessos pelas veias safenas ou femorais.

\section{PACIENTES E MÉTODOS}

No período dejaneiro 1995 a dezembro 1999, foram implantados 586 CV CLP em crianças com câncer no Serviço de Cirurgia Pediátrica O ncológica do Instituto $\mathrm{N}$ acional de Câncer - Rio de Janeiro - Brasil.

Q uanto ao sítio de implantação dos CVCLP, foram $536(91 \%)$ no braço, 45 (8\%) no tórax e 5 (1\%) em outros locais.

Sítio de escolha de implantação dos CVCLP: 0 braço foi nossa primeira opção para inserção de CVCLP, por considerarmos vários benefícios em relação ao tórax: 1 - mais seguro, poisquando o cateter estáativado, permite uma ótima fixação do CVCLP ao sistema de hidratação, evitando que uma tração brusca acidental comprometa 0 funcionamento do cateter, o que não acontece em relação à fixação quando este é colocado no tórax (Figura 1); 2 - mais confortável, pois permite todos os movimentos do paciente, sem restrições, mesmo com o CVCLP "ativado" (Figura 1); 3 - o paciente não necessita assistir o manuseio do seu cateter, o que impossível no CVCLP instalado no tórax, o que diminui sensivelmente o trauma emocional e a ansiedade da manipulação do cateter (Figura 2); 4 - é mais discreto e esteticamente menos visível, uma vez que fica localizado na face anterior do braço, fator importante, principalmente em pacientes adolescentes (figura 3).

T écnica: 0 procedimento de implantação dos CV CLP ocorreu sempre na sala de cirurgia, sob anestesia geral inalatória, combinada com anestesia local com lidocaína a $1 \%$. 0 acesso preferencial ao sistema venoso foram sempre as veias braquiais, abordadas através de incisão transversa na região anterior do braço, por flebotomia, em RN e lactentes. Às vezes optamos pelas veias axilares, devido ao calibre, pois necessitamos introduzir na luz 
da veia um cateter com um calibre não menor do que 4,5 fr, para permitir um bom fluxo, infusões de hemoderivados e refluxo, para coleta de sangue, sem maiores dificuldades e riscos de obstrução.

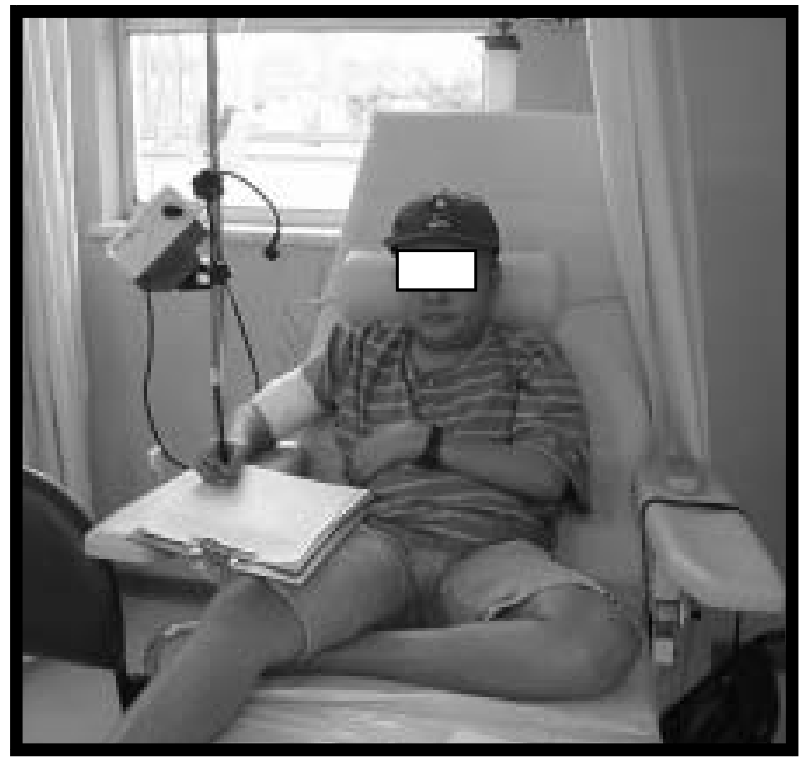

Figura 1. Total mobilidade do paciente com segurança.

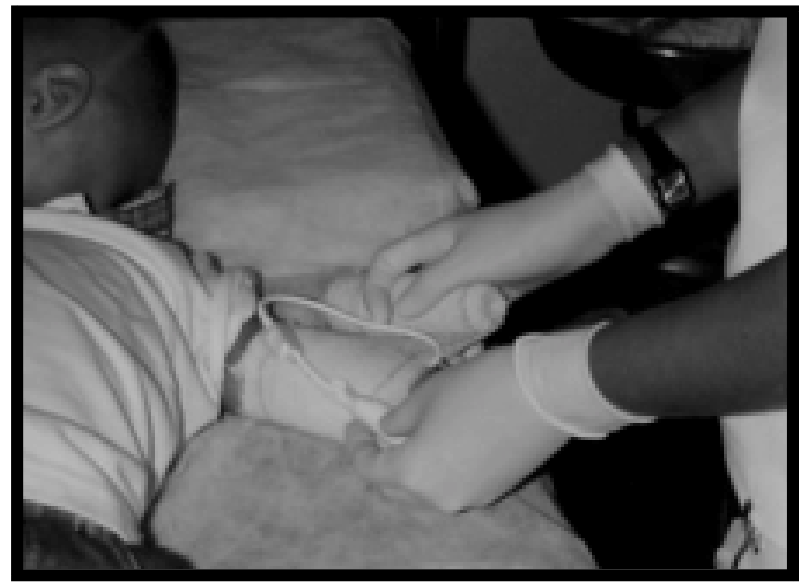

Figura 2. Curativo envolvendo todo o CVCLP e manipulação sem a participação do paciente.

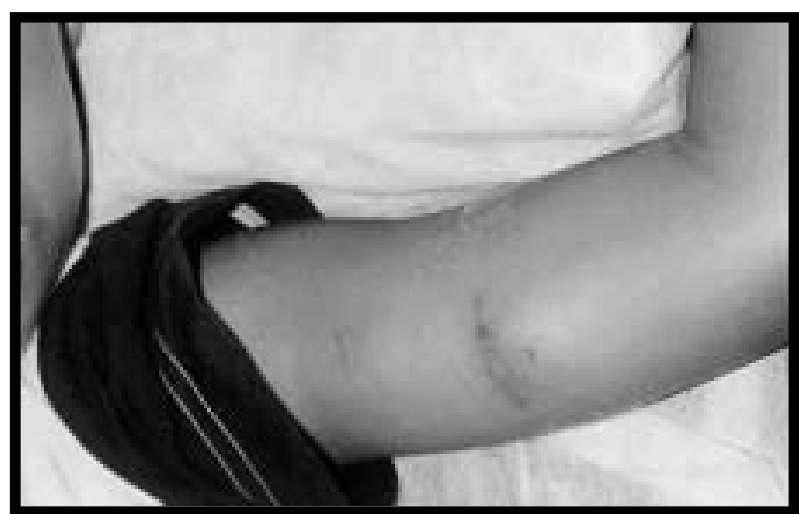

Figura 3. Esteticamente bem discreto.
0 cateter venoso central de longa permanência semiimplantado (CVCLPSI) foi exteriorizado por contraincisão, distante, da primeira incisão, pelo menos $2 \mathrm{~cm}$, ambas na face anterior do braço. Q uando optamos pelo cateter venoso central de longa permanência totalmente implantado (CVCLPTI), o reservatório foi posicionado há aproximadamente $2 \mathrm{~cm}$ da incisão da dissecção venosa, através de uma bolsa confeccionada no tecido subcutâneo, sendo o reservatório ancorado à fáscia profunda por pelo menos dois pontos de fio inabsorvível, para não haver a possibilidade de rotação do mesmo. É necessário que o reservatório seja proporcional ao tamanho do braço da criança, para isso é importante termos reservatórios de vários tamanhos (Figura 4).

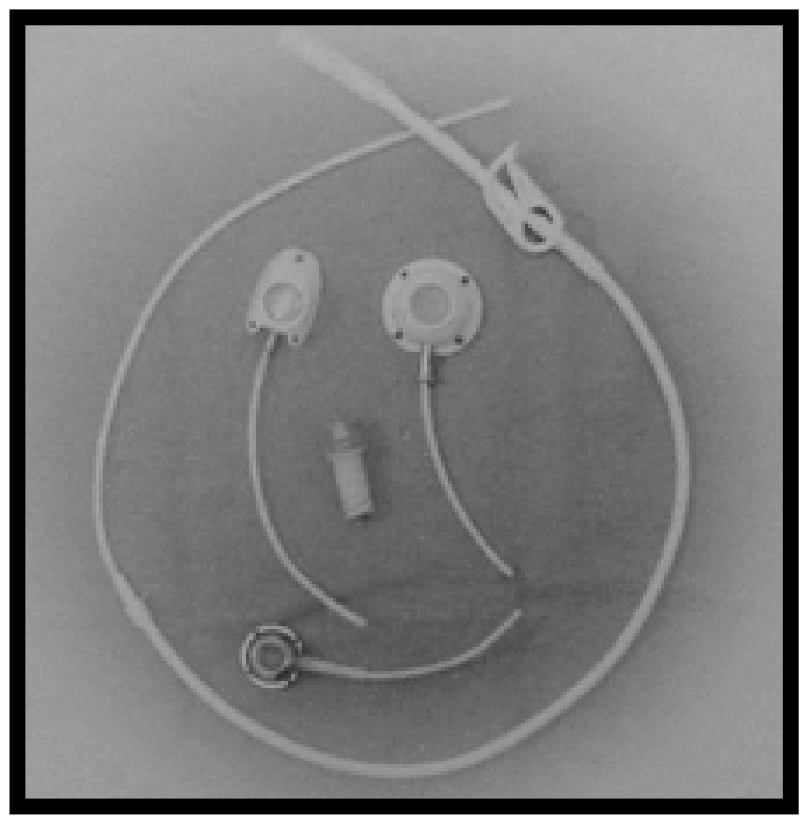

Figura 4. Para cada tamanho de paciente um reservatório adequado.

Foi de suma importância a avaliação da posição da extremidade distal do cateter no per-operatória por radioscopia, pois nem sempre conseguimos na primeira vez situá-lo na posição ideal, ou seja, no 1/3 médio da veia cava superior, algumas vezes, o cateter se posicionou na veia jugular interna, ou progrediu para a veia subclávia contra-lateral. M anobras de apoio, tais como lateralização da cabeça para o lado do braço envolvido, compressão dos territórios supraclaviculares e da fúrcula esternal, mostram-se úteis nessas situações, para posicionar o cateter no local ideal.

U ma vez posicionado, realizamos a fixação do reservatório à aponeurose com fio inabsorvível 3-0, no caso dos CVCLPTI, ou a fixação do cateter à pele, no caso dos CVCLPSI, também com fio inabsorvível 3-0. 
Realizamos em seguida a heparinização do cateter com $2 \mathrm{ml}$ de uma solução de $1 \mathrm{ml}$ de soro fisiológico com $500 \mathrm{UI}$ de heparina, volume suficiente para preencher toda a luz de ambos os tipos de CVCLP.

0 curativo é oclusivo, envolvendo-se com gaze a parte externa do CVCLPSI e cobrindo-o com uma atadura de crepom ao redor do braço (Figura 2), devendo ser trocado no dia seguinte, da colocação, no "Ambulatório de Cateteres", local no nosso hospital exclusivo para a realização de todos os procedimentos relativos aos CVCLP. ${ }^{7}$

Este tipo de curativo é utilizado também quando 0 cateter está ativado, sendo uma das vantagens da implantação do CVCLP no braço, pois permite ao paciente realizar todos os movimentos do membro, sem risco de acidentes, uma vez que é quase impossível ocorrer a desconexão do CVCLP ao sistema de hidratação, o que seria uma complicação bastante grave quando se trata de quimioterápico, infundido no subcutâneo, ou mesmo de "arrancamento" por tração acidental do cateter. Esta complicação grave é comum em paciente pediátrico, como ocorre com uma certa freqüência com os dispositivos implantados no tórax, já que o curativo nesta região, é de difícil fixação, o que não ocorre no braço.

N os curativos só utilizamos gaze e crepon, não há necessidade de adesivos (esparadrapo e outros) para fixação dos cateteres, com isto evitamos o contato direto com a pele do paciente, os quais são utilizados nos cateteres implantados no tórax, que com freqüência causam dermatite de contato com o uso constante, principalmente em nosso país, cujas temperaturas elevadas propiciam uma maior sudorese, além de não serem seguros quando o CV C LP estiver ativado, soltando com relativa facilidade, podendo provocar acidentes.

0 período para ativação do CVCLP vai depender do tipo de cateter utilizado. No CVCLPSI pode ser utilizado imediatamente após a colocação, já no CVCLPTI é recomendado aguardar 72 horas para utilizá-lo, evitando-se assim a dor da punção num local recentemente traumatizado cirurgicamente, que geralmente apresenta um certo grau de edema da pele devido à confecção da bolsa no subcutânea para alojamento do reservatório.

Em casos especiais, quando há necessidade de um acesso venoso imediatamente após a colocação do CVCLPTI, o cateter deverá ser puncionado na sala de operação, com o paciente ainda sob o efeito anestésico.

\section{RESULTADOS}

No período de janeiro 1995 a junho 1999, foram implantados 586 CV C LP em crianças com câncer pelo
Serviço de Cirurgia Pediátrica O ncológica do Instituto N acional de Câncer, dos quais 536 (91\%) CVCLP no braço, $45(8 \%)$ no tórax e $5(1 \%)$ em outros locais (Figura 1). Indicamos os cateteres semi-implantados em 438 pacientes $(75 \%)$, em pacientes pré-escolares e escolares e os cateteres totalmente implantados em 148 pacientes $(25 \%)$, geralmente lactentes e adolescentes.

Apesar de serem os CV CLP um grande avanço em termos de qualidade no tratamento oncológico, eles não estão livres de complicações, muito pelo contrário, elas são freqüentes, principalmenteem mãos não experientes. A quantidade de complicações está diretamente relacionada à experiência do cirurgião no procedimento e dos profissionais que vão manipulálo. $M$ as apesar da experiência e dos cuidados na manipulação, as complicações existem e a mais freqüente é a infecção (Figura 5). N os 586 dispositivos implantados no nosso serviço, encontramos 175 episódios de infecção (29\%) sendo os que tiveram sua etiologia fúngica foram retirados imediatamente, enquanto que os de origem bacteriana foram tratados e permaneceram no paciente quando a infecção foi controlada em 72 horas, caso contrário foram retirados. A Comissão de Controle da Infecção $\mathrm{H}$ ospitalar $(\mathrm{CCIH})$ do hospital, no período de janeiro de 1995 a julho 2001, num total de 745 cateteres venosos centrais de longa permanência estudados relata uma taxa de infecção global de 2,51 / 1000 dias de cateter, com uma taxa de 1,50 / 1000 dias $(p \varangle 0,05)$ para os CVCLPTI e uma taxa de 3,06 / 1000 dias de cateter $(p<0,05)$ para os CVCLPSI, sendo que nos pacientes com doenças malignas hematológicas a taxa foi de 4,88 / $1000(P<0,05)$ e para os pacientes com tumores sólidos foi de 1,82 / $1000(p \varangle 0,05)$, sendo 0 tempo médio para aparecimento do primeiro episódio de 124 dias. $^{18}$

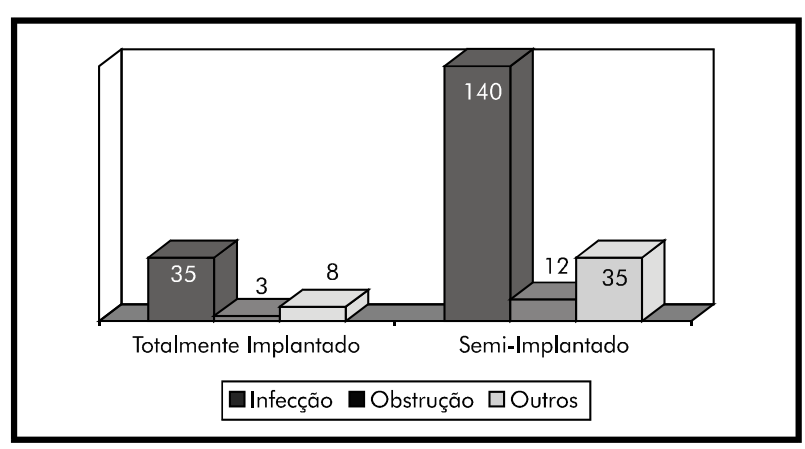

Figura 5. Complicações dos CVCLP

As complicações mecânicas apresentaram-se num percentual bem menor, 7,41\% para os CVCLPTI e de 10,7\% para os CVCLPSI e estão descritas na tabela 1. 
Tabela 1. Complicações mecânicas

\begin{tabular}{l|c|c}
\hline \multicolumn{1}{c|}{ Complicação } & CVCLPTI & CVCLPSI \\
\hline Exteriorização do reservatório & 3 & 0 \\
\hline Exteriorização do anel de fixação cuff & 0 & 15 \\
\hline Ruptura do cateter & 2 & 8 \\
\hline Má posição da extremidade do cateter & 0 & 8 \\
\hline Desconexão do cateter ao reservatório & 1 & 0 \\
\hline Saída espontânea do cateter & 0 & 1 \\
\hline Rotação do reservatório & 1 & 0 \\
\hline Fratura do reservatório de plástico & 1 & 0 \\
\hline Obstrução do cateter & 3 & 12 \\
\hline Total & $11(7,4 \%)^{*}$ & $47(10,7 \%)^{* *}$ \\
\hline
\end{tabular}

*Percentual referente ao total de CVCLPTI implantados (11/148)

** Percentual referente ao total de CVCLPSI implantados (47/438)

Em relação à trombose venosa profunda tivemos apenas 3 casos $(0,5 \%)$, bem menor do que os dados relatados na literatura. ${ }^{21,22}$

C abeobservar quenenhuma complicação encontrada pode ser relacionada diretamente ao sítio de implantação no braço.

0 tempo de permanência dos 586 cateteres foi avaliado em dias, conforme figura 6 . Todos os CVCLP foram retirados até 30 dias após sua colocação (54 cateteres - 9\%). 0 motivo da sua retirada foi a infecção bacteriana resistente a antibióticoterapia ou fúngica, ambas situações a opção é a retirada do cateter. 0 desconhecimento do paradeiro de 9 cateteres $(2 \%)$ se deve ao óbito do paciente, não ter relação com o CVCLP, ou seja, faleceram com o cateter funcionando. (Figura 6)
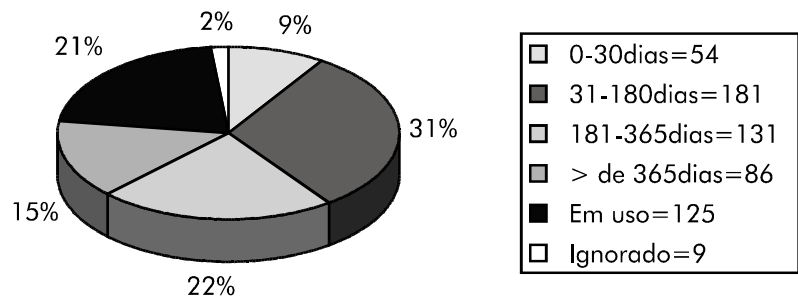

Figura 6. Tempo de permanência dos CVCLP

\section{DISCUSSÃO}

0 acesso venoso central de longa permanência tem sido largamente realizado através da punção das veias subclávias, jugulares interna e externa, pela técnica de Seldinger e variações, ${ }^{2,3,8-16}$ posicionando o reservatório ou exteriorizando, nos cateteres semi-implantados, na parede torácica anterior, e menos freqüentemente por dissecção venosa seguida de flebotomia. ${ }^{3,16,17}$

O utros sítios de inserção são descritos como o braço3 e a coxa10, mas sempre como condutas de exceção em casos particulares. Muito embora a literatura seja extensa, em relação ao uso, indicações e sítios de implantação, são escassos os trabalhos que relatam 0 membro superior como via de escolha para a colocação de CVCLP na infância. ${ }^{3-6}$

Carreira et al. fazem uso do braço como via alternativa, tendo implantado 41 Ports entre 617 CV CLP neste local (7\%), com taxa de infecção geral de $8 \%{ }^{4}$

Em 1994, Johnson et al relataram 61 casos de CVCTI tipo PAS-Port implantados no antebraço de crianças e adultos, com bons resultados: meia-vida livre de eventos de 278 dias, com taxas de complicação de $13,1 \%$, e de infecção igual a $6,6 \%$. ${ }^{6}$ Lundberg et al., em 1995, descreveram taxas de infecção bastante baixas, 0,08 / 100 dias de cateter para ports implantados no braço, em crianças. ${ }^{5} \mathrm{H}$ ills et al., em 1997, reportaram a maior série relatada na literatura até hoje, 100 CVCLPTI implantados no braço, com 0,038 complicações / 100 dias de cateter, e 0,029 / 100 dias de cateter, por infecção. Sua taxa de sucesso a longo prazo foi de $93,8 \%$. $^{3}$

O s autores que descrevem o braço como local de implantação, o fazem através de flebotomia, ${ }^{3-6,17}$ assim como nós, por acharmos ser a forma mais segura de acessá-las. As punções ficam para as veias subclávia ou jugular quando utilizadas.

Consideramos essencial que o procedimento seja realizado em Centro Cirúrgico, sob anestesia geral e com radioscopia per-operatória, pois a incidência de posicionamento inadequado da extremidade do cateter, 
na primeira avaliação radioscópica é bastante freqüente, ${ }^{3,4,9,12-14} \mathrm{C}$ il na sua experiência com 20 pacientes refere a utilização da radioscopia como elemento de sucesso para o implante dos cateteres,20 apesar de Kincaid et al relatarem sucesso no posicionamento da extremidade do cateter em posição central, sem controle fluoroscópico, em $92 \%$ dos pacientes $(558 / 559) .{ }^{15}$

A trombose venosa profunda tem sido cada vez mais objeto de estudo na faixa etária pediátrica, na nossa série apenas 3 casos foram encontrados. D oria et al. sugerem que esta entidade é pouco diagnosticada pela carência de sintomas e por ser pouco suspeitada, refere ainda o CV C como fator predisponente para tal, o que nós concordamos. $M$ ale et al corroboram 0 acima citado ressaltando a maior incidência no lado esquerdo, acesso pela veia subclávia e a inserção por punção, como fatores que contribuem para eventos trombóticos. ${ }^{22}$

\section{CONCLUSÃO}

0 acesso venoso central é crucial para o manejo do paciente com câncer não só para a administração de drogas, como hemoderivados, antibióticos, nutrição parenteral e coleta de sangue. ${ }^{19} \mathrm{~N}$ os pacientes pediátricos portadores de CVCLP observamos uma melhor qualidade de vida e maior adesão ao tratamento prolongado. Acreditamos que a implantação de CVCLP no braço, deva ser a primeira opção em crianças que necessitem de acesso venoso central de longa permanência.

Q uando ativado, a fixação do CV CLP localizado no braço, é muito mais fácil e seguro, pois está protegido da tração acidental, comum em crianças, permitindo um gotejamento regular, assim como total mobilidade do paciente, além de evitar as dermatites causados pelos adesivos, quando implantado no tórax.

$Q$ uando inativado, o curativo do cateter venoso central de longa permanência semi-implantado se torna simples, seguro e confortável, uma vez que é apenas uma atadura ao redor do braço. Já o cateter venoso central de longa permanência totalmente implantado, quando fora de uso, fica praticamente imperceptível, uma vez que não utiliza curativo e as cicatrizes cirúrgicas e o reservatório localizam-se na face anterior do braço, ficando pouco visível, devido à posição anatômica do mesmo, o que permite, principalmente aos adolescentes uma maior sociabilidade, fato extremamente importante nestes pacientes oncológicos.

D as complicações dos CV CLP encontradas no nosso levantamento nenhuma dela teve relação com a localização no braço.

\section{REFERÊNCIAS}

1. Aubaniac R. L'injection intraveineuse sous-claviculaire. Presse M ed. 1952;60:1656-9.

2. Johnson EM, Saltzman D A, Suh G, AdamsRA, Leonard AS. Complications and risks of central venous catheter placement in children. Surgery. 1998;124(5):911-6.

3. HillsJR, CardellaJ F, CardellaK, Waybill PN . Experience with 100 consecutivecentral venousaccessarm portsplaced by interventional radiologists. J Vasc Interv Radiol. 1997 N ov-D ec;8(6):983-9.

4. CarreiraVillamor JM, Reyes Perez R, Pulido-D uqueJM, Gorriz Gomez E, Pardo M D, Argiles Vives JM, et al. Percutaneousimplant of $\mathrm{H}$ ickman cathetersand reservoirs: long-term experience. Rev Clin Esp. 1997 N ov;197(11):740-4.

5. Lundberg G, Wahlberg E, Rickberg A, O lofsson P. PASPort: a new implantable vascular access device for arm placement: experiencesfrom thefirst two years. Eur J Surg. $1995 \mathrm{M}$ ay;161(5):323-6.

6. Johnson JA, DidlakeRH . Peripherally-placed central venous access ports: clinical and laboratory observations. Am Surg. 1994 D ec;60(12):915-9.

7. Gonçalves AR, M ariño CH V, Carvalho RV, Bach RVW, Finóquio R. Ambulatório para pacientes portadores de cateter venoso central delonga permanência: experiência de 5 anos [tema livre]. $20^{\circ}$ Congresso B rasileiro de Cirurgia Pediátrica; 1999 out 10-14; Recife.

8. Snyder CL, Saltzman D A, Leonard AS. Central venous accessin infantsand small children: anew technique.JPEN . 1990;14:662-3.

9. Franceschi $D$, Specht M A, Farrel C. Implantablevenousaccess device. J Cardiovasc Surg (Torino). 1989;30(1):124-9.

10. M athur M N , Storey D W, W hite G H , Ramsey-Stewart G. Percutaneousinsertion of long-term venous access catheters via the external iliac vein. Aust N Z J Surg. 1993 N ov;63(11):858-63.

11. Pullyblank AM , Carey PD, PearceSZ, Tanner AG, G uillou $P J, M$ onson JR. Comparison between peripherally implanted ports and externally sited catheters for longterm venous access. Ann R Coll Surg Engl. 1994 Jan;76(1):33-8.

12. Biffi R, Corrado F, deBraud F, de LuciaF, ScarpaD, Testori $A$, et al. Long term, totally implantablecentral venous access ports connected to a $G$ roshong catheter for chemotherapy of solid tumours: experiencefrom 178 cases using a single type of device. Eur J Cancer. 1997 Jul;33(8):1190-4.

13. Biffi R, deBraud F, O rsi F, Pozzi S, M auri S, Goldhirsch A, et al. Totally implantable central venous acess ports for longterm chemotherapy. A prospective study analyzing complications and costs of 333 devices with a minimum follow-up of 180 days. Ann 0 ncol. 1998 Jul;9(7):767-73.

14. Crowley J, Pereira J K, H arris LS, Becker CJ. Radiologic 
placement of long-term subcutaneousvenousaccess portsin children. AJR AmJ Roentgenol. 1998 Jul;171(1):257-60.

15. Kincaid EH , D avis PW, Chang M C, Fenstermaker JM , Pennell TC. "Blind" placement of long-term central venous access devices: report of 589 consecutive procedures. Am Surg. 1999 Jun;65(6):520-3; discussion 523-4.

16. M unro FD , G illet PM, Wratten JC, Shaw M P, T homasA, $M$ acKinlay $G A$, et al. Totally implantablecentral venous access devicesfor paediatric oncology patients. M ed Pediatr Oncol.1999;33:377-81.

17. Petersen C, Fuchs J, Kotzur A, Strauss G. Central venous indwelling catheter systemsin pediatriconcology from thesurgical viewpoint. Klin Padiatr. 1998 M ar-Apr;210(2):65-9.

18. Schimer M, Velasco E, M artins CAS, D iasLM, Gonçalves VM SC, VicuñaCH , et al. Incidencerates of bloodstream infections in children with long-term central venous cathetersat Instituto N acional deC âncer, Brazil. Int J Infect Dis. 2002;6 Suppl 2:2S-29.

19. ShuklaN K, D asD K, D eo SV, RainaV. N a analysis of longterm venous access catheters in cancer patiens; experience from a tertiary care centrein India. J Postgrad M ed. 2002 Jan-M ar;48(1):21-4.

20. Cil BE. Radiological placement of chest portsin pediatric oncology patients. Eur Radiol. 2004 N ov;14(11):2015-9.

21. D oria S, N oguchi DT, Paccez JD. Trommbose venosa profunda na faixa etária pediátrica. Rev BrasTer Intensiva. 2001 jan-mar;13(1):15-20.

22. $M$ aleC, $C$ hait $P, A$ Andrew $M, H$ annaK, Julian J, $M$ itchell L; PARKAA Investigators. Central venous line-related thrombosis in children: association with central venousline location and insertion technique. Blood. 2003 Jun 1;101(11):4273-8. 